ISSN: 0210-1696

DOI: http://dx.doi.org/10.14201/scero20154632746

\title{
ANÁLISIS DE LA FORMACIÓN \\ UNIVERSITARIA RECIBIDA POR LOS \\ TITULADOS UNIVERSITARIOS ESPAÑOLES \\ CON DISCAPACIDAD AUDITIVA EN LA \\ ADQUISICIÓN DE LAS COMPETENCIAS \\ PROFESIONALES
}

\section{Analysis of Undergraduate Training Given to Students with Hearing Impairment in their Acquisition of Professional Competences}

\author{
Mariona Dalmau Montalà \\ Departamento de Psicología. Facultad de Psicología. Ciencias de la Educación y del Deporte \\ Blanquerna. Universidad Ramon Llull. Barcelona. C/ Císter, 34. 08022 Barcelona \\ marionadm@blanquerna.url.edu \\ Ingrid SALA BARS \\ Departamento de Psicología. Facultad de Psicología. Ciencias de la Educación y del Deporte \\ Blanquerna. Universidad Ramon Llull. Barcelona \\ Montserrat Llinares Fité \\ Departamento de Psicología. Facultad de Psicología. Ciencias de la Educación y del Deporte \\ Blanquerna. Universidad Ramon Llull. Barcelona
}

Recepción: 18 de diciembre de 2014

Fecha de aceptación definitiva: 16 de julio de 2015

Biblid. [0210-1696 (2015) vol. 46 (3), n. ${ }^{\circ} 255$, julio-septiembre; 27-46]

Resumen: La finalidad de este estudio ha sido conocer la percepción de los titulados universitarios españoles con discapacidad auditiva sobre su formación universitaria. La metodología utilizada ha sido cuantitativa-cualitativa y descriptiva. La muestra participante se configuró a partir de 84 titulados universitarios españoles con discapacidad auditiva. Los resultados permiten afirmar que los titulados universitarios con discapacidad 
auditiva, generalmente, no reciben en la universidad los apoyos adecuados para poder desarrollar, suficientemente, las competencias profesionales; estos titulados creen que los empresarios consideran imprescindible tener un buen dominio de todas las competencias profesionales; por otra parte, estos graduados manifiestan que las prácticas durante sus estudios universitarios son una buena oportunidad para su inserción laboral; finalmente, se pone de relieve que entre el profesorado universitario existe un importante desconocimiento de las necesidades y dificultades de los estudiantes con discapacidad auditiva.

Palabras Clave: educación superior; prácticas en la educación universitaria; competencias profesionales; titulados universitarios con discapacidad auditiva; inserción laboral.

AвSTRACT: The aim of this study was to understand how university graduates with a hearing impairment in Spain perceived their studies. The method used was quantitativequalitative and descriptive. The participating sample consisted of 84 Spanish university graduates with a hearing impairment. The results allow us to confirm that: university graduates with a hearing impairment, in general, are not provided with appropriate/ sufficient support at university to develop their professional competence; these graduates believe that employers consider good command of professional competences to be pivotal; professionalizing practices during university studies are an opportunity for the employment of graduates with hear impairment; finally, it is highlighted that university lecturers lack knowledge of the needs and difficulties of students with hearing impairment.

KEYS WORDS: higher education; higher education practices; professional competences; students with a hearing impairment; employability.

\section{Introducción}

$\mathrm{E}$

L ESTUDIO QUE PRESENTAMOS SURGIÓ A PARTIR DE UNA INVESTIGACIÓN más amplia titulada La Integración laboral de los universitarios españoles con discapacidad. Detección de las fortalezas y las debilidades en el momento del acceso al mercado laboral español: Percepción de los universitarios y percepción de las empresas (Dalmau, Llinares, Sala y Giné, 2010), financiada en su totalidad por Fundación UNIVERSIA. El objetivo principal de dicha investigación era conocer la percepción de los titulados universitarios españoles con discapacidad sobre su educación superior universitaria e inserción laboral. A la luz de los resultados obtenidos en esta investigación consideramos importante conocer con más detalle las características específicas en base al tipo de discapacidad de los titulados universitarios. Concretamente, en este artículo hacemos referencia al estudio que analiza la percepción de los titulados universitarios españoles con discapacidad auditiva sobre los apoyos necesarios durante sus estudios universitarios para desarrollar las competencias profesionalizadoras, las prácticas durante los estudios universitarios y, finalmente, las competencias profesionalizadoras más valoradas por los empresarios. 


\section{Antecedentes}

La Constitución española (1978) sienta las bases para reconocer los derechos de los ciudadanos españoles, pero es con la LISMI (1982) que empiezan a hacerse efectivas las políticas de integración educativa, motivo por el cual se empieza a constatar el acceso a los estudios universitarios y a la obtención del título por parte de personas con discapacidad, con lo que se abre paso al mercado laboral competitivo.

Concretamente en el ámbito laboral de las personas con discapacidad destacar el artículo 35 de la Constitución española (1978): “Todos los españoles tienen el deber de trabajar y el derecho al trabajo, a la libre elección de profesión u oficio, a la promoción a través del trabajo y a una remuneración suficiente para satisfacer sus necesidades y las de su familia”. En nuestro país la inserción laboral de las personas con cualquier tipo de discapacidad toma relevancia a partir de la entrada en vigor de la Ley de Integración Social del Minusválido (1982). Por su parte las Naciones Unidas (2006) en el artículo 27 de la Convención sobre los Derechos de las Personas con Discapacidad también reconocen el derecho de estas personas al trabajo en igualdad de condiciones con las demás personas.

El periodo de transición a la vida adulta, lo que conlleva en muchas ocasiones la inserción en el mundo laboral, es desalentador para muchos jóvenes y sobre todo para aquellos que tienen discapacidad (Johnson, Stodden, Emanuel, Luecking y Mack, 2002; Osgood, Foster, Flanagan y Ruth, 2005; Wagner, Newman, Cameto, Levine y Marder, 2007). Una de las aportaciones importantes para solventar esta situación ha sido la introducción del trabajo con apoyo. Se trata de un modelo de integración laboral para las personas con discapacidad que tiene como objetivo promover el empleo en el contexto ordinario. Así, Verdugo y Jordán de Urríes (2002) consideran el "empleo con apoyo" como el que está integrado en la comunidad, dentro de empresas normalizadas, para personas con discapacidad que tradicionalmente no han tenido acceso al mercado laboral; y en condiciones lo más similares posible en trabajo y sueldo a las de otro trabajador sin discapacidad. El empleo con apoyo responde a unos principios formulados por Mank (1998) que son:

... - El trabajo tiene significado en nuestra vida.

- Las personas con discapacidades significativas pueden trabajar.

- Integración e inclusión son preferibles a segregación y exclusión.

- La elección y la satisfacción son valiosas.

- Desarrollo de carrera profesional, no sólo de empleos.

- Creación de apoyos individuales, no de programas.

- Énfasis en la calidad de vida... (pp. 7-8).

En esta misma línea, Bellver (2002) entiende el empleo con apoyo como el trabajo remunerado en empresas ordinarias, con un contrato laboral individual y para personas con discapacidad que necesitan apoyo y seguimiento; el sistema de apoyo que debe ir retirándose a medida que el trabajador es autosuficiente; el que hace posible la igualdad de oportunidades al aplicarse en entornos integrados; aquel que primero 
coloca a la persona con discapacidad en la empresa y después se forma; el apoyo que es natural y lo deciden los participantes; y, por último, aquellas oportunidades de empleo a las personas que se consideran no aptas para trabajar en los talleres especiales. En el contexto español existen diferentes estudios que ponen de relieve la práctica basada en este tipo de trabajo como son: la inserción laboral normalizada con apoyo de personas con discapacidad intelectual (Alomar, 2004); la inserción laboral mediante el modelo de trabajo con apoyo (Serra, 2004); las personas con discapacidad psíquica contratadas por la administración de la Generalitat de Catalunya (Rius, 2005); la calidad de vida de las personas con discapacidad intelectual en los Centros Ocupacionales y Centros especiales de trabajo (García, 2008). El Real Decreto 870/2007 regula el empleo con apoyo.

Existen distintas investigaciones a nivel internacional (Stodden y Dowrick, 1999; Kennedy y Harris, 2005; Coleman, 2008) y nacional (Oliver, Sancho y Galiana, 2012; Dalmau, Llinares y Sala, 2013; Rodríguez, Suso, Vázquez y Velasco, 2013) que ponen en evidencia la importancia que tiene para las personas, con discapacidad en general, obtener un título o una formación universitaria como elemento de ayuda para el acceso al mundo laboral. Esta formación es determinante cuando contempla la diversidad de todo el estudiantado y presta especial atención a las personas con discapacidad. Este planteamiento queda recogido en el nuevo Marco Europeo de la Educación Superior y en la Ley 51/2003 donde se introducen las definiciones sobre "accesibilidad universal” y “diseño para todos”. Más adelante el Real Decreto 1393/2007, modificado por el Real Decreto 861/2010, promueve la aplicación en todos los programas educativos, incluidos los universitarios, de la accesibilidad universal y el diseño para todos. Esta normativa genera un marco idóneo para la aplicación del Diseño Universal en el ámbito universitario que garantiza una educación de calidad para todos (Dalmau, Guasch, Sala, Llinares, Dotras, Álvarez y Giné, 2015).

Los trabajos que se relacionan a continuación hacen referencia de forma específica a titulados universitarios con discapacidad: la integración laboral de los titulados universitarios con gran discapacidad física (Vilà y Pallisera, 2002), la superación de las barreras psicosociales para la integración de los titulados universitarios (Campoy y Pantoja, 2003), las barreras físicas y psicosociales en el proceso de la inserción laboral de los universitarios con discapacidad visual (Polo, 2006), integración laboral de los universitarios españoles con discapacidad (Dalmau, Llinares, Sala y Giné, 2010), calidad de la empleabilidad en universitarios con discapacidad (Oliver, Sancho y Galiana, 2012) y discapacidad, estudios superiores y mercado de trabajo. Barreras de acceso y repercusión en la inserción laboral (Rodríguez, Suso, Vázquez y Velasco, 2013).

En el trabajo de Vilà y Pallisera (2002) sobre integración laboral de personas con titulación universitaria y discapacidad se focaliza la función prioritaria de la educación superior en la profesionalización, y en conseguir que los docentes universitarios aseguren que los estudiantes con discapacidad desarrollen estrategias que posibiliten la consecución de los aprendizajes básicos. Las prácticas externas y las experiencias laborales que de ellas se derivan son un componente esencial de los aprendizajes universitarios profesionalizadores. En este mismo estudio, las autoras consideran que para favorecer la inserción de los titulados universitarios con discapacidad en el mercado laboral son esenciales: las habilidades que facilitan la independencia de la persona, las 
habilidades de carácter sociopersonal (autoconocimiento, autoestima, responsabilidad, iniciativa, toma de decisiones, seguridad en uno mismo, relaciones interpersonales, etc.) y la dimensión laboral. Todos estos requisitos coinciden plenamente con lo que se considera competencias profesionales (González y Wagenaar, 2003) de los titulados universitarios españoles en general (UNIVERSIA, ACCENTURE y F. TELEFÓNICA, 2008). En este estudio comparativo entre la opinión de los empresarios, las universidades y los estudiantes se concluye que por parte de los empresarios las competencias más valoradas son la motivación, la capacidad de adaptación y la capacidad de relación con los demás. También ponen de manifiesto que otras competencias como la autonomía, la negociación y el liderazgo se pueden desarrollar durante la trayectoria profesional en el seno de la empresa. Por parte de las universidades las competencias más valoradas son, en primer lugar, las de tipo actitudinal como la motivación y la capacidad de adaptación y, en segundo lugar, la expresión lingüística, la autonomía, la negociación y el liderazgo. Y, por último, los estudiantes valoran las competencias destacando las cognitivas y las instrumentales por encima de las demás. En cambio, consideran que las de tipo actitudinal se desarrollan a partir del entorno familiar y de la iniciativa individual. Para la adquisición de las competencias profesionales es importante que los estudiantes universitarios con discapacidad cuenten con los apoyos necesarios para garantizar su desarrollo y así facilitar su inserción en el mundo laboral (Dalmau, Llinares y Sala, 2013).

En relación a la inserción laboral de las personas con discapacidad se han explorado distintos ámbitos como la influencia del tipo de discapacidad para la obtención de un puesto de trabajo (Bordieri, Drehmer y Taylor, 1997; Villa, 2003), los factores que inciden en los procesos de inserción laboral (Jordán de Urríes y Verdugo, 2003; Pallisera, Fullana y Vilà, 2005). Villa (2003) indica que en la población española las personas con discapacidad auditiva son las que tienen un índice de actividad laboral más elevado y una menor tasa de desempleo.

En el ámbito universitario y, concretamente, en relación a los estudiantes con discapacidad auditiva, los autores Crespo (2009), Durán y Miguélez (2009) y Jáudenes (2009) expresan que la clave para el acceso al trabajo y a la educación superior empiezan en los tramos educativos anteriores. En concreto con la formación del profesorado y con la dotación de los apoyos necesarios durante la trayectoria educativa incluida la formación universitaria. Asimismo ponen énfasis en este tipo de formación como elemento facilitador de la inserción laboral.

Con la finalidad de contribuir a un mayor conocimiento y difusión de la experiencia en la educación universitaria de las personas con discapacidad auditiva en relación al desarrollo de las competencias profesionales que les facilite la inserción laboral, nos hemos planteado los objetivos que presentamos a continuación.

\section{Objetivos}

El objetivo general que desarrollamos en este estudio es el siguiente: conocer la percepción de los titulados universitarios españoles con discapacidad auditiva sobre su formación universitaria. 
Los objetivos específicos son los siguientes:

a) Conocer la experiencia de los titulados universitarios con discapacidad auditiva sobre los apoyos recibidos durante su educación universitaria para desarrollar las competencias profesionales.

b) Averiguar, a partir de la experiencia de los titulados universitarios con discapacidad auditiva, cuáles son las competencias profesionales más valoradas por los empresarios.

c) Identificar el valor de las prácticas durante el desarrollo de la educación universitaria en base a la adquisición de las competencias profesionales.

d) Detectar los aspectos que pueden contribuir a la mejora de la formación universitaria de los estudiantes con discapacidad auditiva.

\section{Método}

La información se ha obtenido a partir de los datos recogidos a través de un cuestionario elaborado ad hoc con preguntas cerradas y abiertas (ver Anexo).

Las respuestas a las preguntas cerradas han sido tratadas informáticamente mediante una hoja de cálculo. Se efectuó un análisis descriptivo de los datos, a partir del cual hemos identificado, en los casos que hemos considerado oportuno, aquellas variables en las cuales sería relevante efectuar un análisis inferencial.

Para este último empleamos la prueba $\chi^{2}$.

El análisis de las respuestas a las preguntas abiertas se ha llevado a cabo a partir de crear un sistema de categorías para cada apartado a analizar. Dada la naturaleza de este estudio, para la elaboración del sistema de categorías se ha seguido el proceso inductivo, es decir, extraer de la información obtenida rasgos concretos y agruparlos según su similitud en base al objeto de estudio. Las categorías elaboradas son exhaustivas y mutuamente excluyentes, cumpliendo así los requisitos propios de todo sistema de categorización (Buendía, Colás y Hernández, 1997).

\section{Participantes}

La muestra participante está compuesta por 84 personas tituladas universitarias con discapacidad auditiva. De estas, 32 son hombres y 52 son mujeres. La media de edad es de 35,63 años. Entre ellas, tal y como muestra el Gráfico 1, quedan representadas las comunidades autónomas siguientes: Andalucía, Aragón, Canarias, Cantabria, Castilla y León, Castilla La Mancha, Cataluña, Comunidad Valenciana, Galicia, Islas Baleares, Madrid, País Vasco y el Principado de Asturias. 


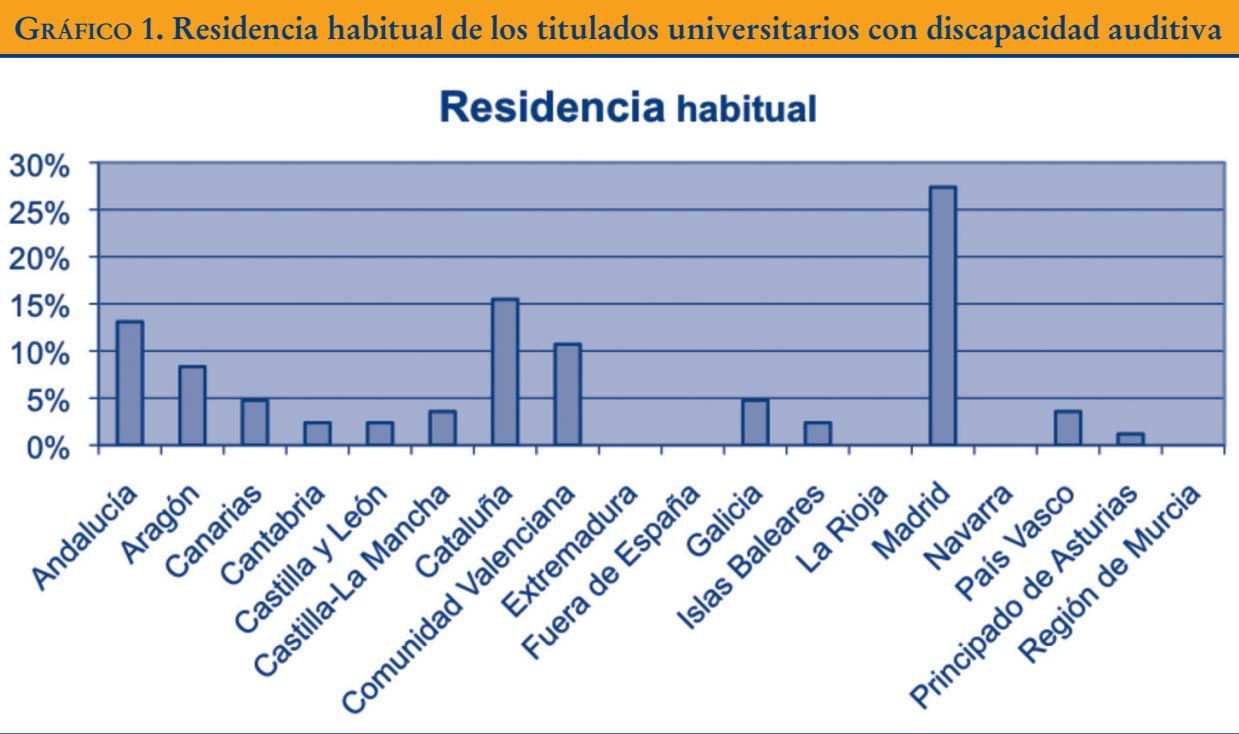

El grado de discapacidad de las personas participantes se sitúa, tal y como se muestra en el Gráfico 2, en el rango $<40 \%$ hasta $79 \%$. La mayoría de las cuales se encuentran en el intervalo $<40 \%$ hasta $49 \%$, lo que representa un $81,71 \%$ de la muestra.

GRÁfICO 2. Grado de discapacidad de los titulados universitarios con discapacidad auditiva

\section{Grado de discapacidad}

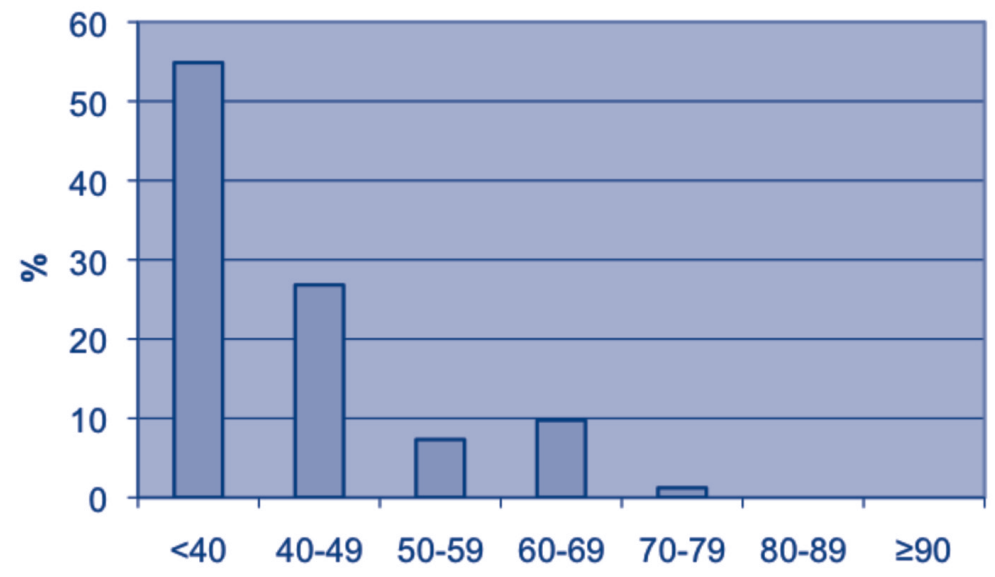

(C) Ediciones Universidad de Salamanca

Siglo Cero, vol. 46 (3), n. ${ }^{\circ} 255,2015$, julio-septiembre, pp. 27-46 
Referente a la rama de estudio (ver Gráfico 3) escogida por este grupo de estudiantes destaca, de manera significativa, la de estudios de ciencias sociales y jurídicas $(60,71 \%)$.

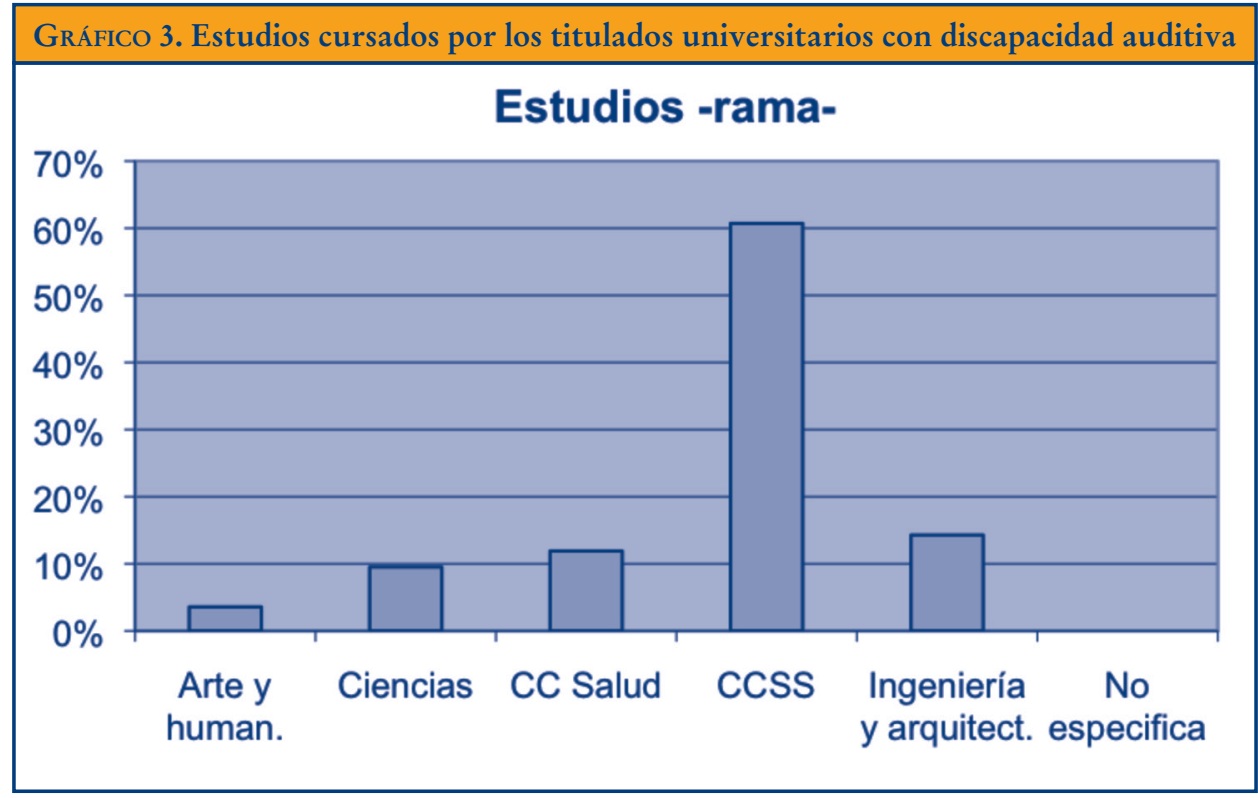

Por la ley de protección de datos (Real Decreto 1720/2007, de 21 de diciembre, por el que se aprueba el Reglamento de desarrollo de la Ley Orgánica 15/1999, de 13 de diciembre, de protección de datos de carácter personal), no podíamos acceder directamente al grupo de personas participantes. Las asociaciones de personas con discapacidad y distintas fundaciones involucradas en la inserción laboral de titulados con discapacidad nos ayudaron a acceder al grupo de titulados universitarios con discapacidad. Las direcciones de dichas asociaciones y fundaciones fueron extraídas de la base de datos del Centro Español de Documentación sobre Discapacidad (CEDD). Este fue el procedimiento para hacer llegar a los titulados universitarios con discapacidad la invitación a participar. Por este motivo, no podemos concretar el número de personas con discapacidad auditiva que han sido invitadas. Solamente podemos referirnos al total de sujetos que aceptaron formar parte del estudio y que nos ha permitido configurar la muestra de titulados universitarios con discapacidad auditiva.

\section{Elaboración del instrumento y recogida de información}

En este apartado exponemos el procedimiento seguido para obtener la información de los participantes. 
Modalidad de recogida de información

Se optó por la modalidad on-line. El programa elegido, tras las consultas pertinentes a expertos informáticos, fue el Google Docs. Este programa permite que los participantes viertan directamente sus respuestas a una hoja de cálculo Excel, lo cual facilita la recogida y clasificación de la información desde un primer momento, así como su posterior tratamiento. A su vez, garantiza el pleno anonimato de los participantes.

Construcción del cuestionario: Elección del contenido. Juicio de expertos. Prueba piloto

Después de revisar la literatura disponible sobre el tema, y de acuerdo con la finalidad y los objetivos del estudio, se diseñaron los apartados del cuestionario con las preguntas correspondientes. Se hicieron varias revisiones hasta considerar que el cuestionario estaba listo para ser sometido a juicio de expertos. A cada uno de los cinco expertos se le explicó, por escrito, la finalidad y los objetivos del estudio y se le pidió su opinión en relación a la adecuación del contenido, la forma y la comprensión del cuestionario. Las valoraciones aportadas por los expertos permitieron modificar algunos aspectos e introducir cambios en el contenido y la forma hasta dar por finalizada la nueva versión del cuestionario, que sería utilizada en la prueba piloto correspondiente. Este fue sometido a una segunda prueba piloto. El análisis del contenido y las sugerencias que acompañaron el cuestionario permitieron la elaboración definitiva del mismo.

\section{Descripción del instrumento}

El cuestionario incluye un apartado introductorio en el que se enmarca y presenta, brevemente, la investigación. A continuación, se pide la colaboración y se garantiza la total confidencialidad de los datos aportados. Tras agradecer la participación, se dan las indicaciones para responder y remitir la información.

La primera parte del cuestionario recoge datos de tipo demográfico. A continuación se indican los apartados: elección de los estudios universitarios; formación universitaria recibida; servicios universitarios de inserción laboral; acceso profesional en el mercado laboral; legislación y administración; concesión de apoyos durante el desarrollo profesional; actitudes y creencias hacia la discapacidad por parte de los empleadores y de los empleados, y, finalmente, un apartado de observaciones.

\section{Requisitos éticos de la investigación}

Para cumplir con los requisitos éticos de la investigación se ha garantizado la confidencialidad de los datos recibidos durante todo el proceso de recogida y de tratamiento de la información. También se ha procurado emplear terminología ética en 
toda la documentación y se ha puesto a disposición de los participantes los resultados del estudio.

\section{Resultados}

A continuación presentamos los resultados obtenidos. Los detallamos a través de los apartados siguientes:

Apoyos necesarios para que los estudiantes con discapacidad auditiva puedan desarrollar a lo largo de su formación universitaria las competencias profesionalizadoras

El 63,10\% de los titulados universitarios con discapacidad auditiva, del estudio que presentamos, cree que el hecho de tener unos estudios universitarios les facilitará la entrada al mercado laboral.

En relación al desarrollo de las competencias profesionalizadoras, estos titulados opinan que las universidades les facilitaron los apoyos necesarios para adquirir las siguientes (ver Gráfico 4): motivación, entusiasmo y ganas de aprender (52,38\%) y preocupación por la calidad, por hacer las cosas bien $(51,19 \%)$. Sin embargo, manifiestan que la universidad no les facilitó de manera suficiente los apoyos necesarios para adquirir las competencias siguientes: dominio de la lengua inglesa $(89,21 \%)$, capacidad de negociación $(71,43 \%)$, potencial de liderazgo $(70,23 \%)$, habilidades interpersonales $(60,72 \%)$, habilidades comunicativas $(60,71 \%)$, iniciativa y espíritu emprendedor $(60,71 \%)$, capacidad para generar nuevas ideas $(59,52 \%)$, manejo del ordenador $(58,33 \%)$, capacidad de adaptarse a nuevas situaciones $(53,57 \%)$, habilidades de búsqueda y gestión de la formación $(53,57 \%$ ) y capacidad de organizar y planificar $(51,19 \%)$. Finalmente, encontramos que el 50\% de los participantes explicita que la universidad les proporcionó los apoyos suficientes para el desarrollo de la habilidad para trabajar de forma autónoma y la capacidad de trabajar bajo presión, frente al $50 \%$ que opina lo contrario.

Competencias profesionales más valoradas por los empresarios según los titulados universitarios con discapacidad auditiva

Los titulados universitarios con discapacidad auditiva, desde su experiencia profesional, mencionan que los empresarios valoran muy positivamente la posesión de las competencias siguientes (ver Gráfico 5): preocupación por la calidad, por hacer las cosas bien (91,67\%); motivación, entusiasmo y ganas de aprender (86,91\%); capacidad para adaptarse a nuevas situaciones $(86,91 \%)$; manejo del ordenador $(86,91 \%)$; habilidades comunicativas $(85,72 \%)$; habilidad para trabajar de forma autónoma $(85,72 \%)$; capacidad de organizar y planificar $(83,34 \%)$; capacidad de trabajar bajo presión (82,96\%); habilidades de búsqueda y gestión de la información (82,14\%); habilidades 
GrÁfICO 4. Grado en que la universidad facilitó a los estudiantes con discapacidad auditiva los apoyos necesarios para desarrollar las competencias profesionales

\section{Teniendo en cuenta su experiencia profesional y su situación de discapacidad indique en qué grado su universidad le facilitó los apoyos necesarios para poder desarrollar las siguientes}

Habilidades comunicativas
Dominio de la lengua inglesa
Habilidad para trabajar
de forma autónoma
v tomar decisiones
Iniciativa y
espíritu emprendedor

Preocupación por la calidad, por hacer las cosas bien

Motivación, entusiasmo y ganas de aprender

Capacidad de organizar y planificar,

saber administrar el tiemno

Capacidad de trabajar bajo presión

Potencial de liderazgo, capacidad de influir $v$ motivar a otros

Capacidad de negociación, saber convencer y aceptar otros buntos de vista

Habilidades interpersonales, saber relacionarse con otros

Capacidad para adaptarse a nuevas situaciones,

flexibilidad

Capacidad para generar nuevas ideas,

creatividad e innovación

Manejo del ordenador

Habilidades de búsqueda y gestión de información

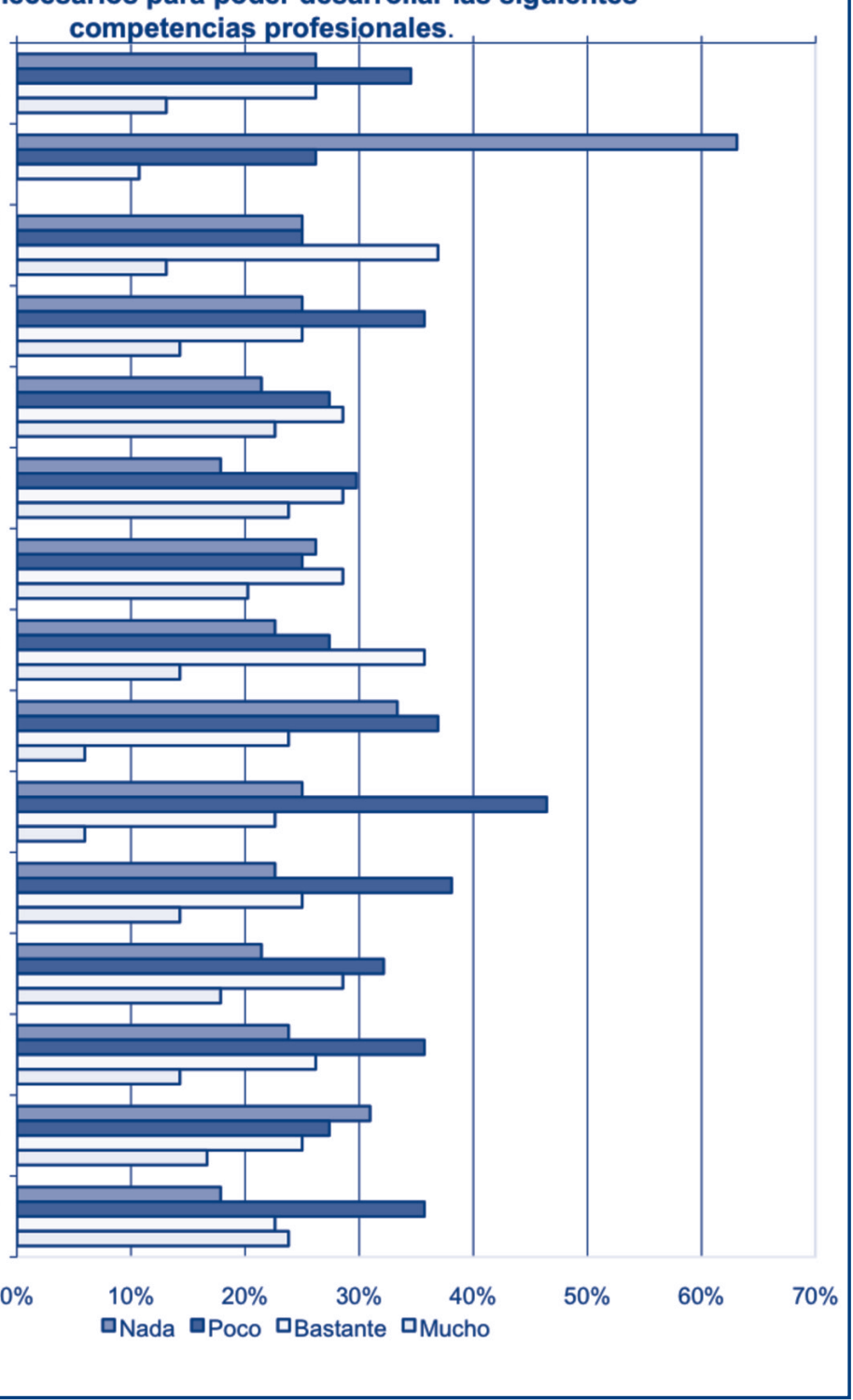

(C) Ediciones Universidad de Salamanca

Siglo Cero, vol. 46 (3), n. ${ }^{\circ}$ 255, 2015, julio-septiembre, pp. 27-46

$$
-37-
$$


interpersonales $(80,96 \%)$; iniciativa y espíritu emprendedor $(78,58 \%)$; capacidad para generar nuevas ideas (78,58\%); capacidad de negociación (77,38\%); potencial de liderazgo $(67,85 \%)$; y dominio de la lengua inglesa $(55,96 \%)$.

Por otra parte, el análisis cualitativo de la información obtenida indica que los titulados universitarios con discapacidad auditiva consideran que los empleadores también valoran los aspectos siguientes:

- La experiencia laboral

- La disponibilidad y lealtad a la empresa

- Los estudios de postgrado

- La invisibilidad de la discapacidad

\section{Desarrollo de las prácticas durante los estudios universitarios}

A partir de su propia experiencia laboral los titulados universitarios con discapacidad auditiva consideran en un $66,67 \%$ que las prácticas realizadas durante sus estudios universitarios han contribuido adecuadamente a su desarrollo profesional, frente a un $33,33 \%$ que no valoran positivamente esta experiencia. Este último grupo echó en falta durante sus prácticas los aspectos siguientes:

- La vinculación entre universidad y empresa, que favoreciera el conocimiento mutuo necesario para el desarrollo de buenas prácticas dirigidas a los estudiantes con discapacidad auditiva.

- Recibir, durante sus prácticas, apoyos específicos adaptados a sus necesidades como estudiantes con discapacidad auditiva.

- Sensibilidad por parte del entorno laboral hacia sus necesidades como personas con discapacidad auditiva.

Finalmente se pone de manifiesto que el 14,28\% del total de la muestra no realizó prácticas durante el desarrollo de sus estudios universitarios. La mayoría de los cuales opina que hubiera sido importante para su formación haber tenido una experiencia en el mundo laboral a través de la realización de prácticas universitarias.

Aportaciones significativas de los titulados universitarios con discapacidad auditiva vinculadas a la mejora de su formación universitaria

El análisis cualitativo de los resultados obtenidos pone de manifiesto que existe, por parte de la universidad en general, un importante desconocimiento de las necesidades y dificultades de las personas con discapacidad auditiva. También nos permite 
Gráfico 5. Grado en que los empleadores valoran el dominio de las competencias profesionales según los titulados universitarios con discapacidad auditiva

\section{Teniendo en cuenta su experiencia profesional y su situación de discapacidad indique en qué grado los EMPLEADORES valoran el dominio de las siguientes competencias profesionales.}

Habilidades comunicativas

Dominio de la lengua inglesa

Habilidad para trabajar de forma autónoma y tomar decisiones

Iniciativa y espiritu emprendedor

Preocupación por la calidad,

por hacer las cosas bien

Motivación, entusiasmo

y ganas de aprender

Capacidad de organizar y planificar,

saber administrar el tiempo

Capacidad de trabajar bajo presión

Potencial de liderazgo, capacidad de influir v motivar a otros

Capacidad de negociación, saber convencer y aceptar otros puntos de vista Habilidades

interpersonales,

saber relacionarse con

Capacidad para adaptarse a

nuevas situaciones.

Capacidad para generar nuevas ideas, creatividad e innovación

Manejo del ordenador

Habilidades de búsqueda y gestión de información

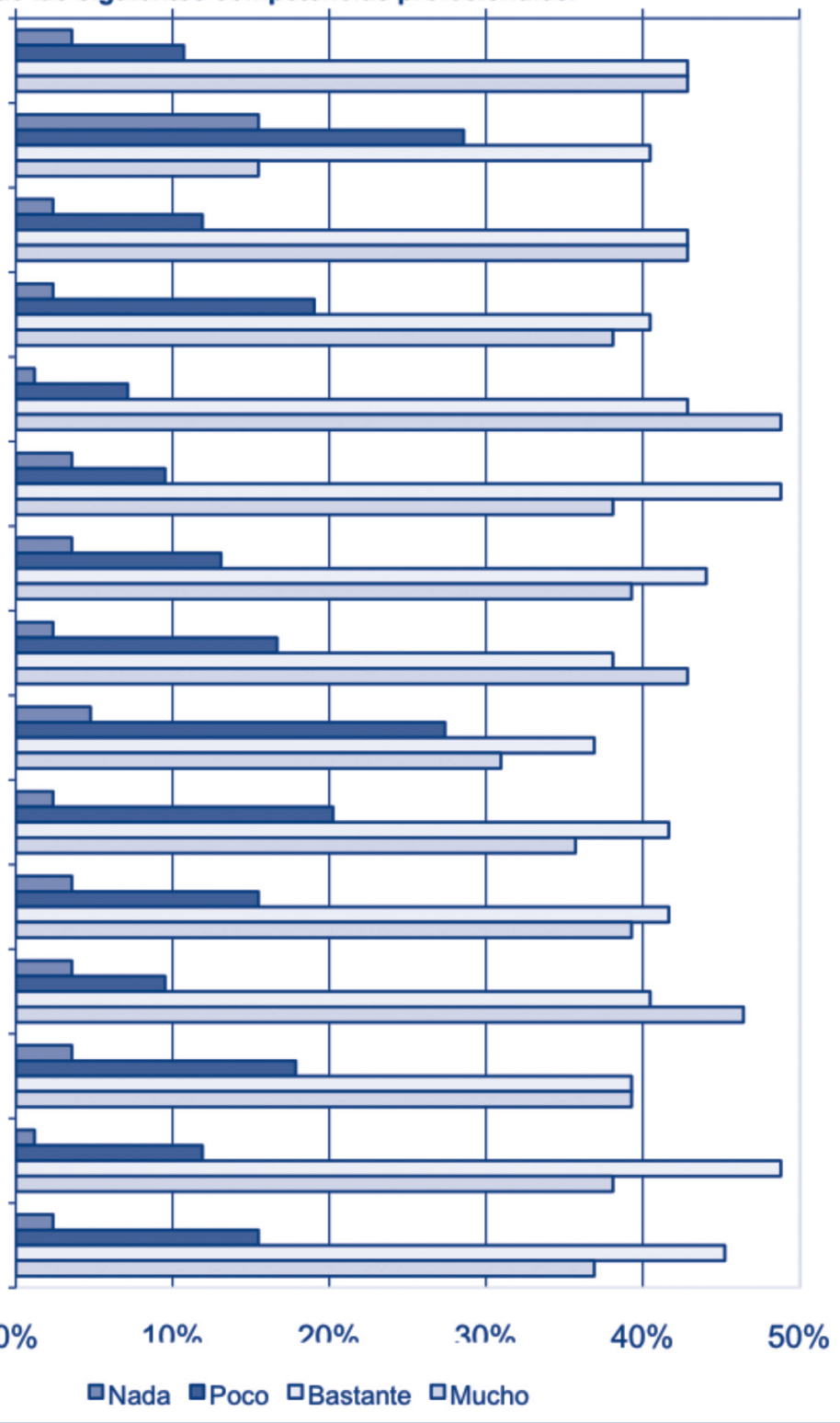

(C) Ediciones Universidad de Salamanca

Siglo Cero, vol. 46 (3), n. ${ }^{\circ} 255,2015$, julio-septiembre, pp. 27-46

$$
-39 \text { - }
$$


exponer aquellos aspectos, considerados relevantes por parte de los titulados con discapacidad auditiva, que según ellos hubiesen contribuido a un mejor desarrollo de sus estudios universitarios. Estos aspectos son los siguientes:

- Respeto y valoración de la diversidad dentro de las aulas universitarias.

- Formación del profesorado universitario acerca de las necesidades y de las dificultades en el aula de los estudiantes universitarios con discapacidad auditiva.

- Conocimiento y dominio, por parte del profesorado, de herramientas que ayuden a desarrollar sus clases de forma inclusiva. En concreto que les permitiese: proporcionar material de clase adaptado (audiovisuales, apuntes, bibliografía...); ofrecer las evaluaciones en diferentes formatos; transmitir la información oral de forma adaptada para los estudiantes con discapacidad auditiva; potenciar la comunicación e interacción conjunta entre estudiantes con y sin discapacidad auditiva.

- Existencia de Servicios universitarios de orientación y de inserción laboral para estudiantes con discapacidad, en concreto para estudiantes con discapacidad auditiva.

\section{Discusión y conclusiones}

En este apartado presentamos la discusión y conclusiones a partir de los resultados obtenidos en la investigación.

La mayoría de los titulados universitarios con discapacidad auditiva de nuestro estudio afirman que tener estudios universitarios facilita el acceso al mundo laboral. Esto coincide con los estudios llevados a cabo por Coleman (2008), Dalmau Llinares y Sala (2013), Kennedy y Harris (2005), Oliver, Sancho y Galiana (2012), Rodríguez, Suso, Vázquez y Velasco (2013) y Stoden y Dowrick (1999), que también manifiestan la importancia para las personas con discapacidad en general de obtener un título universitario. Por lo tanto, podemos afirmar que la obtención de unos estudios universitarios se considera facilitador para el acceso al mercado laboral entre los titulados universitarios con discapacidad auditiva.

Crespo (2009), Durán y Miguélez (2009) y Jáudenes (2009) ponen de relieve el valor que tienen los apoyos para que los estudiantes con discapacidad auditiva desarrollen con éxito los estudios universitarios. En este sentido los participantes de la presente investigación manifiestan que solo recibieron los apoyos necesarios para adquirir dos de las catorce competencias profesionalizadoras establecidas por el proyecto Tuning Educational Structures in Europe (González y Wagenaar, 2003) y UNIVERSIA, ACCENTURE Y F. TELEFONICA (2008). Estas dos competencias son motivación, entusiasmo y ganas de aprender y preocupación por la calidad, por hacer las cosas bien. Por lo que se puede concluir que la universidad no facilita, de manera suficiente, a los estudiantes con discapacidad auditiva, los apoyos necesarios para 
poder adquirir la mayoría de las competencias profesionales. De esta conclusión se desprende la necesidad de que el profesorado universitario esté formado en temas de accesibilidad universal y metodologías que favorezcan, desde un modelo inclusivo, la calidad del proceso de enseñanza-aprendizaje de las personas con discapacidad tal y como afirman Dalmau, Guasch, Sala, Llinares, Dotras, Álvarez y Giné (2015), Sala (2013) y Sala, Sánchez, Diez y Giné (2014).

La experiencia laboral de los titulados universitarios con discapacidad auditiva reafirma la necesidad de tener desarrolladas las competencias profesionales valoradas por los empresarios. Estos apuntan que los empresarios valoran muy positivamente el domino de las competencias profesionalizadoras establecidas por el proyecto Tuning Educational Structures in Europe (González y Wagenaar, 2003), además de la experiencia laboral, la disponibilidad y la lealtad a la empresa, los estudios de posgrado y la invisibilidad de la discapacidad. El estudio realizado por Rodríguez, Suso, Vázquez y Velasco (2013) pone de relieve la reticencia de los empresarios a contratar a titulados con discapacidad por la falta de información sobre la realidad de la discapacidad. No obstante, Dalmau, Llinares y Sala (2013) manifiestan que una vez los empresarios han vivido la experiencia de contratar un titulado universitarios con discapacidad su percepción cambia positivamente. Todo esto refleja que todavía prevalecen los estereotipos entre el empresariado sobre las capacidades de las persona con discapacidad, pero si se trabaja a favor de la inclusión laboral estos estereotipos desaparecen.

Los resultados obtenidos en nuestro trabajo indican que las prácticas en las empresas son muy valoradas por la mayoría de los titulados con discapacidad auditiva. Pero también se ha detectado un porcentaje de estos titulados con experiencias menos positivas por echar de menos la relación entre la universidad y la empresa, la falta de apoyos específicos y la escasa sensibilidad por parte del entorno laboral hacia las necesidades de las personas con discapacidad auditiva. Todo ello coincide con los resultados (Rodríguez, Suso, Vázquez y Velasco, 2013) que consideran que las personas con discapacidad han encontrado verdaderos problemas para realizar las prácticas ya sea por el rechazo de los empresarios al no incluirlos en estos programas. En sintonía con esta realidad Oliver, Sancho y Galiana (2012) consideran importante impulsar recursos para integrar la relación entre la universidad, las empresas y otras entidades con la finalidad de potenciar el conocimiento mutuo y así romper las ideas preconcebidas.

Las funciones de los servicios específicos de orientación preuniversitaria y universitaria para estudiantes con discapacidad deberían favorecer la elección de unos estudios ajustados a la realidad de cada estudiante, acompañarle y proporcionarle los recursos que faciliten su inserción laboral. Las universidades deben disponer de servicios de atención, orientación y empleo preparados para atender las necesidades específicas de los titulados universitarios con discapacidad auditiva. En este sentido también apuntan Rodríguez, Suso, Vázquez y Velasco (2013) la necesidad de potenciar en los centros universitarios la figura de un mediador laboral con una formación concreta en el ámbito de la discapacidad y en el empresarial. 


\section{Propuestas de mejora}

A partir del estudio presentado planteamos algunas propuestas con la finalidad de contribuir a la mejora de la educación universitaria de los estudiantes con discapacidad auditiva y su profesionalización. Estas propuestas se presentan ordenadas en tres apartados de acuerdo al contexto al que se dirigen:

\section{A) Profesorado universitario}

Proponemos diseñar planes de formación y acción para el profesorado universitario orientados a proporcionar el conocimiento adecuado para dar respuesta a las necesidades de la diversidad de todos los estudiantes universitarios. Esta formación debería facilitar:

- El conocimiento de los principios del Diseño Universal y su aplicación en el aprendizaje universitario (Diseño para Todos del Aprendizaje).

- La implementación del Diseño para Todos del Aprendizaje (cambio de perspectiva y enfoque del proceso de enseñanza-aprendizaje y de la evaluación en la universidad).

- El conocimiento y la incorporación en el aula de los apoyos humanos y tecnológicos necesarios para que los estudiantes con discapacidad auditiva puedan desarrollar sus estudios universitarios.

- El conocimiento de la existencia de las fundaciones y asociaciones vinculadas a la discapacidad auditiva y sus líneas de acción.

\section{B) Institución universitaria}

Planteamos concienciar a la institución universitaria de la importancia de su compromiso con la formación de los estudiantes universitarios con discapacidad, y en concreto de las personas con discapacidad auditiva. Este compromiso podría explicitarse entre otras acciones a partir de:

- Facilitar servicios de apoyo a los docentes universitarios para poder implementar con éxito el Diseño para Todos del Aprendizaje en sus aulas.

- Potenciar servicios de apoyo al estudiante universitario con responsables altamente preparados para atender las necesidades de los estudiantes universitarios con discapacidad auditiva.

- Incrementar la vinculación entre la universidad y las empresas para facilitar el desarrollo de las prácticas durante los estudios universitarios de las personas con discapacidad, y en concreto con discapacidad auditiva. Esta vinculación 
debería favorecer, por parte de la empresa, una mayor aceptación de las personas con discapacidad como futuros profesionales, permitiendo así ver las potencialidades por encima de las dificultades. Además, esta vinculación debería representar una tutorización compartida (universidad-empresa) de las prácticas para asegurar su accesibilidad y su adaptación.

- Contar con las fundaciones y asociaciones de personas con discapacidad auditiva para el asesoramiento en temas de formación, adaptación y accesibilidad del entorno universitario así como del entorno de prácticas y de salidas laborales.

\section{C) Etapas educativas anteriores a la formación universitaria}

Proponemos sensibilizar al profesorado y orientadores de todas las etapas educativas anteriores a la formación universitaria sobre la importancia de la intervención docente en cada una de estas etapas para que los alumnos con discapacidad auditiva puedan acceder y desarrollar con éxito sus estudios universitarios. Esta sensibilización debería orientarse a que los maestros, profesores y orientadores:

- Desarrollaran expectativas positivas respecto al futuro profesional de los alumnos con discapacidad auditiva; reconociendo los estudios universitarios como un importante factor facilitador para el acceso al mercado laboral ordinario de estos alumnos.

- Tomaran consciencia de la importancia de algunos de los aprendizajes iniciales como base para el éxito en el desarrollo de las competencias profesionales a lo largo de la educación universitaria, en concreto: la comprensión lectora, la expresión oral y escrita, las relaciones interpersonales y la adquisición y dominio de la lengua inglesa.

- Conocieran e implementaran los principios del Diseño Universal y su aplicación en el aprendizaje (Diseño para Todos del Aprendizaje).

- Acompañaran a los estudiantes con discapacidad auditiva, a lo largo de su formación, ayudándoles a descubrir sus capacidades, habilidades y posibilidades en el contexto en el que se estén desarrollando, para que sean los propios estudiantes los que puedan decidir su itinerario educativo y profesional.

\section{Limitaciones del estudio}

Al finalizar nuestro estudio queremos dejar constancia de las limitaciones que hemos detectado a lo largo de la investigación y que creemos necesario tener en cuenta en el momento de generalizar los resultados. Por una parte, desconocemos el número total de titulados universitarios con discapacidad auditiva españoles por lo que no podemos conocer el alcance de la representatividad de la muestra real. Otra limitación 
importante ha sido la escasez de investigaciones sobre el tema concreto de la inserción laboral de los titulados universitarios con discapacidad en entornos laborales ordinarios, lo que nos ha impedido contrastar ampliamente los resultados de nuestro estudio con los de otras investigaciones similares. Finalmente, hubiera sido interesante incorporar en el estudio un grupo de estudiantes universitarios sin discapacidad que permitiese un estudio comparativo para investigar la percepción de cada grupo en relación a su formación universitaria. A partir de las limitaciones expuestas entendemos que nuestro trabajo tiene la importancia y el valor de un primer estudio exploratorio sobre el estado actual del tema. Por este motivo consideramos que las propuestas de intervención global que hemos expuesto deben entenderse como un punto de partida hacia nuevas vías de investigación que permitieran desarrollar propuestas de intervención más concretas.

\section{Referencias bibliográficas}

Alomar, E. (2004). El treball dels joves amb retard mental en entorns normalitzats: anàlisi d'una realitat d'un treball amb suport (Tesis doctoral). Recuperada de: http://www.tdx.cat/ TDX-0301107-102903.

Bellver, F. (2002). Perspectivas del empleo con apoyo en España. Real Patronato sobre Discapacidad. En AA. VV., Empleo privado de las personas con discapacidad (pp. 109-118). Madrid: Real Patronato sobre Discapacidad.

Bordieri, J. E., Drehmer, D. E. y Taylor, D. W. (1997). Work life for employees with disabilities: Recommendations for promotion. Rebabilitation Counseling Bulletin, 40, 181-191.

Buendía, L., Colás, P. y Hernández, F. (Eds.) (1997). Métodos de Investigación en Psicopedagogía. Madrid: McGraw-Hill.

Campoy, T. J. y Pantoja, A. (2003). Transición al mundo laboral de alumnos universitarios con discapacidades físicas y sensoriales. Revista de Educación Especial, 33, 39-57.

Coleman, B. (2008). Transition from College to Work: Lived Employment Experiences and Perceptions of College Seniors and recent College Graduates with Physical Disabilities Seeking Employment Opportunities. (Tesis doctoral inédita). The Faculty of the Graduate School Education and Human Development of the George Washington University, Washington DC.

Crespo, C. (2009). Síntesis y reflexión final panel III. FIAPAS, 130, 90-91.

Dalmau, M., Guasch, D., Sala, I., Llinares, M., Dotras, P., Álvarez, M. H. y Giné, C. (2015). Diseño Universal para la Instrucción (DUI). Indicadores para su implementación en el ámbito universitario. Barcelona: Universitat Ramon Llull y Universitat Politècnica de Catalunya.

Dalmau, M., Llinares, M. y Sala, I. (2013). Formación universitaria e inserción laboral. Titulados españoles con discapacidad y competencias profesionalizadoras. Revista Española de Discapacidad, 1 (2), 95-118.

Dalmau, M., Llinares, M., Sala, I. y Giné, G. (2010). Integración laboral de los universitarios españoles con discapacidad. Detección de las fortalezas y debilidades en el momento del acceso al mercado laboral español. Percepción de los universitarios y percepción de las empresas. Madrid: Fundación Universia. 
Durán, A. y Miguélez, V. (2009). Estudiantes con discapacidad auditiva en la Universidad Complutense de Madrid: Análisis de los programas de apoyo y sus resultados obtenidos. FIAPAS, 130, 82-85.

España. Constitución Española. Boletín Oficial del Estado, 29 de diciembre de 1978, núm. 311, 29313-29424.

España. Ley 13/1982, de 7 de abril, de integración social de los minusválidos. Boletín Oficial del Estado, 30 de abril de 1982, núm. 103, 11106-11112.

EsPaÑA. Ley 51/2003, de 2 de diciembre, de igualdad de oportunidades, no discriminación y accesibilidad universal de las personas con discapacidad. Boletin Oficial del Estado, 3 de diciembre de 2003, núm. 289, 43187-43195.

España. Real Decreto 870/2007, de 2 de julio, por el que se regula el programa de empleo con apoyo como medida de fomento de empleo de personas con discapacidad en el mercado ordinario de trabajo. Boletín Oficial del Estado, 14 de julio de 2007, núm. 168, 30618-30622.

España. Real Decreto 1720/2007, de 21 de diciembre, por el que se aprueba el Reglamento de desarrollo de la Ley Orgánica 15/1999, de 13 de diciembre, de protección de datos de carácter personal. Boletín Oficial del Estado, 19 de enero de 2008, núm. 17, 4103-4136.

EsPaña. Real Decreto 1393/2007 de 29 de octubre, por el que se establece la Ordenación de las Enseñanzas Universitarias. Boletín Oficial del Estado, 30 de octubre de 2007, núm. 260, 44037-44048.

EsPañA. Real Decreto 861/2010, de 2 de julio, por el que se modifica el Real Decreto 1393/2007, de 29 de octubre, por el que se establece la ordenación de las enseñanzas universitarias oficiales. Boletín Oficial del Estado, 3 de julio de 2010, núm. 161, 58454-58468.

García, M. (2008). Avaluació de la qualitat de vida de lespersones amb discapacitat intel-lectual en els Centres Ocupacionals $i$ Centres Especials de Treball (Tesis doctoral). Recuperada de: http://www.tdx.cat/TDX-0111108-102847.

GonzÁLEZ, J. y WagenAAR, R. (Eds.) (2003). Tuning Educational structures in Europe. Informe final. Bilbao: Universidad de Deusto.

JÁUDENES, C. (2009). Estudio FIAPAS sobre Inserción Laboral de personas sordas. FIAPAS, $130,86-89$.

Johnson, D. R., Stodden, R., Emanuel, E., Luecking, R. y Mack, M. (2002). Current challenges facing secondary education and transition services. Exceptional Children, 68 (4), 519-531.

Jordán de Urríes, F. B. y Verdugo, M. Á. (2003). El empleo con apoyo en España. Análisis de variables que determinan la obtención y mejora de resultados en el desarrollo de servicios. Madrid: Centro Español de Documentación sobre Discapacidad.

Kennedy, R. B. y Harris, N. K. (2005). Employing persons with severe disabilities: Much work remains to be done. Journal of Employment Counseling, 42 (3), 133-139.

Mank, D. (1998). Valores y empleo para las personas con discapacidad. Siglo Cero, 29 (4), 5-10.

Naciones Unidas (2006). Convención sobre los derechos de las personas con discapacidad y protocolo facultativo. Recuperado de: http://www.un.org/disabilities/documents/convention/convoptprot-s.pdf.

Oliver, A., Sancho, P. y Galiana, L. (2012). Calidad de la empleabilidad en universitarios con discapacidad. Revista Iberoamericana de Psicología y Salud, 3 (2), 109-123.

Osgood, W., Foster, E. M., Flanagan, C. A. y Ruth, G. (Eds.) (2005). On your Own Without a Net: The Transition to Adulthood for Vulnerable Populations. Chicago, IL: University of Chicago Press.

Pallisera, M., Fullana, J. y Vilà, M. (2005). La inserción laboral de personas con discapacidad. Desarrollo de tres investigaciones acerca de los factores favorecedores de los procesos de inserción. Revista de Investigación Educativa, 23, 295-313. 
Polo, M. T. (2006). Barreras físicas y psicosociales en el proceso de inserción laboral de los universitarios discapacitados visuales. En M. A. Cifuentes, M. Fernández y J. A. Gómez (Coords.), La Accesibilidad como medio para educar en la diversidad. Educación, diversidad y accesibilidad en el entorno europeo (pp. 365-370). Burgos: Universidad de Burgos.

Rius, M. (2005). Recerca sobre les persones amb discapacitat psiquica contractades a l'Administració de la Generalitat de Catalunya. Anàlisi de la incidència de la inserció laboral en diferents dimensions de la vida dels treballadors amb discapacitat psiquica (Tesis doctoral). Recuperada de: http://www.tdx.cat/TDX-0110106-140213.

Rodríguez, P., Suso, A., Vázquez, D. y Velasco, M. L. (2013). Discapacidad, estudios superiores y mercado de trabajo. Barreras de acceso y repercusión en la inserción laboral. Recuperado de: http://www.fundaciononce.es/sites/default/files/Discapacidad_estudios_superiores.pdf.

Sala, I. (2013). Universitat i discapacitat. Construint un model d'aula inclusiva en el marc universitari (Tesis doctoral). Recuperada de: http://hdl.handle.net/10803/119739.

Sala, I., Sánchez, S., Díez, E. y Giné, C. (2014). Análisis de los distintos enfoques del paradigma del diseño universal aplicado a la educación. Revista Latinoamericana de Educación Inclusiva, 8 (1), 143-152.

SERRA, F. (2004). La presència del suport natural en els processos d'inclusió laboral mitjançant el model de treball amb suport (Tesis doctoral). Recuperada de: http://www.tdx.cat/bitstream/handle/10803/9386/tfsb1de1.pdf;jsessionid=9512C1ECA46601DA7F0187A4 3C796881.tdx1 ?sequence $=1$.

Stodden, R. A. y Dowrick, P. W. (2001). Postsecondary education and employment of adults with disabilities. American Rehabilitation, 25 (3), 19-23.

Universia, Accenture y Fundación Telefónica (2008). Las competencias profesionales en los estudiantes preuniversitarios. Madrid: ACCENTURE.

Verdugo, M. Á. y Jordán de URríes, F. B. (2002). Investigación sobre características del empleo con apoyo y resultados en diferentes variables. En M. Á. Verdugo y F. B. Jordán de URRíEs (Coords.), Hacia la integración plena mediante el empleo (pp. 51-68). Salamanca, España: Publicaciones INICO.

Vilà, M. y Pallisera, M. (2002). La integración sociolaboral de personas con discapacidad y formación superior. Revista Educación Especial, 33, 51-71.

Villa, N. (2003). Situación laboral de las personas con discapacidad en España. Revista Complutense de Educación, 14, 393-424.

Wagner, M., Newman, L., Cameto, R., Levine, P. y Marder, C. (2007). Perceptions and expectations of youth with disabilities. A special topic report of findings from the national longitudinal transition study-2 (NLTS2). Menlo Park, CA: SRI International. 